\title{
Performance of immature and adult cats on the Hamilton search test
}

\author{
J. M. WARREN AND HELEN B. WARREN \\ THE PENNSYLVANIA STATE UNIVERSITY
}

Nine groups of cats differing in age, breed, past experience on learning tasks, and neurological status were tested on Hamilton's multiple choice test. No significant intergroup differences were obtained, suggesting that the Hamilton search test is of little or no value for investigating either the ontogeny or phylogeny of learning. The finding that normal cats and cats with lesions in the frontal association cortex do not differ in the efficiency of their searching behavior confirms results previously obtained from monkeys.

Harlow (1959) tested immature and adult rhesus monkeys in the WGTA on a modification of Hamilton's (1911, 1916) insoluble multiple choice problem and found that 12- and 20-month old monkeys made many more repetitive errors than a group of monkeys aged 50 months. The extremely slow maturation of adult proficiency in search behavior demonstrated in Harlow's monkeys, together with Hamilton's results, which indicated a trend toward superior performance as a function of increasing age in humans, monkeys, cats and dogs, suggested that Hamilton's search test might provide a useful technique for investigating the ontogeny of learning in subprimate mammals. The purpose of this experiment was to assess the utility of Hamilton's multiple choice test by comparing the search behavior of cats differing in age, breed, previous testing experience, and neurological status.

Method

Subjects. The nine groups of cats studied, comprising a total of $102 \mathrm{Ss}$, are listed in Table 1. The "adult" groups consisted of animals ranging in age from 1-8 $\mathrm{yr}$. The adult mongrel groups were highly sophisticated animals which had been tested for several years on various learning problems in the WGTA (Warren, 1964); the younger groups of mongrels and the two groups of Siamese had previously been tested only upon serial reversals of a spatial discrimination in a Grice box (Warren \& Warren, in press).

Table 1. Total Responses on Hamilton Search Test

\begin{tabular}{lccccc} 
Age & Breed & Lesion & N & Mean & S.D. \\
\hline 2 mos. & Mongrel & - & 15 & 342 & 32 \\
3 mos. & Mongrel & - & 14 & 318 & 31 \\
4 mos. & Mongrel & - & 14 & 323 & 33 \\
5 mos. & Mongrel & - & 11 & 339 & 33 \\
7 mos. & Mongrel & - & 24 & 328 & 41 \\
7 mos. & Siamese & - & 4 & 328 & 26 \\
Adult & Mongrel & - & 5 & 326 & 40 \\
Adult & Mongrel & Frontal & 6 & 338 & 37 \\
Adult & Siamese & - & 9 & 307 & 24 \\
\hline
\end{tabular}

Apparatus and Procedure. The apparatus, illustrated in Warren (1959), consists of four visually identical goal boxes, equidistant from the start box. Only one box contains food on a given trial, and the locus of the food is changed randomly from trial to trial, with the sole restriction that the same box is never reinforced on any two consecutive trials. Adaptation and testing procedures, as well as the methods of analyzing the data, were identical with those described by Hamilton. A trial is a sequence of responses terminated when $S$ finds the food reward. After initial adaptation to the experimental situation, each $S$ is tested 10 trials a day for 10 days, and performance on the series of 100 trials is described in terms of the total number of responses (entries into specific goal boxes), and of types of sequential responses made in searching for the reinforced compartment. The cats, tested under low (1-23 hr.) levels of deprivation, were rewarded with $1 / 2$ in. cubes of pork kidney for correct responses. Results

The results are summarized in Table 1 which shows mean total responses and standard deviation for each group of cats. Analyses of variance confirm the obvious impression that none of the meaningful intergroup comparisons approaches statistical significance. Age is not a significant source of variation among the groups of mongrel $(F<1)$ or Siamese cats $(F=1.77)$. The Siamese and mongrel adults' scores do not differ significantly $(F=1.12)$, and the means for the 7 -month ald mongrel and Siamese cats are identical. The difference between the performance scores of the intact mongrels and those with lesions in the frontal association cortex is also nonsignificant ( $F<1$ ).

As would be expected from the essentially identical means for the various groups, there was no significant variation among the groups with respect to the frequency of repetitive errors, the frequency of systematic, stereotyped searching patterns, or with respect to any other aspect of performance.

\section{Discussion}

Hamilton's "classical" results were based upon very small samples; for example, he studied only three adult cats and two 63-day old kittens. It is obvious from the data presented in Table 1 that his claim that performance on his search task is correlated with the maturational status of cats is not confirmed when a more adequate number of cases is studied.

The results of the present experiment are also rather difficult to reconcile with the notion that per- 
formance on the Hamilton search test is correlated with phyletic status. The scores of individual cats ranged from 232 to 436 in this study. The mean scores for almost every group of Ss studied by Hamilton, except normal human adults, falls within this range, suggesting that the distributions of intra- and interspecies differences in performance by animals are almost equivalent. Thus, there is little or no evidence that the version of Hamilton's search task used in this experiment provides a valid means for studying either the ontogeny or phylogeny of learning.

Multiple differences in apparatus, response requirements, and procedural variables prevent any unequivocal interpretation of the discrepancy between the results obtained from cats and those obtained from monkeys by Harlow. Our failure to obtain a significant difference in search behavior between cats deprived of the frontal association cortex and their intact controls is, however, completely compatible with the results obtained in studies with monkeys subjected to removals of the homologous cortex (Meyer \& Settlage, 1958; Harlow, Akert, \& Schiltz, 1964).

\section{References}

Hamilton, G. V. A study of trial and error reactions in mammals. J. anim. Behav., 1911, 1, 33-66.

Hamilton, G. V. A study of perseverance reactions in primates and rodents. Behav. Monogr., 1916, 3, No. 13, 1-65.

Harlow, H. F. The development of learning in the rhesus monkey. Scient. American, 1959, 47, 459-479.

Harlow, H. F., Akert, K., \& Schiltz, K. A. The effects of bilateral prefrontal lesi ans on learned behavior of neonatal, infant and preadol escent monkeys. In J. M. Warren \& K. Akert (Ed.), The frontal granular cortex and behavior. New York: McGraw-Hill, 1964. Pp. 126-148.

Meyer, D. R., \& Settlage, P. H. Analysis of simple searching behavior in the frontal monkey. J. comp. physiol. Psychol., 1958, $51,408-410$.

Warren, J. M. Perseverative reactions in chicks and kittens. $J$. Psychol., 1959, 47, 9-12.

Warren, J. M. The behavior of carnivores and primates with lesions in the prefrontal cortex. In J. M. Warren \& K. Akert(Eds.), The frontal granular cortex and behavior. New York: MeGraw-Hill, 1964. Pp. 168-191.

Warren, J. M., \& Warren, H. B. Spatial reversal learning by kittens. J. comp. physiol. Psychol., (in press).

\section{Note}

1. This research was supported by grant MH 04726 from the National Institute of Mental Health, U. S. Public Health Service. 